\title{
THE MEAN SQUARE OF THE LOGARITHM OF THE ZETA-FUNCTION
}

\author{
MICHEL BALAZARD \\ Laboratoire d'Algorithmique Arithmétique (CNRS), Mathématiques, Université de Bordeaux 1, \\ 351, cours de la Libération, 33405 Talence, France \\ e-mail:balazard@math.u-bordeaux.fr \\ and ALEKSANDAR IVIĆ \\ Katedra Matematike RGF-a, Universiteta u Beogradu, Djusina 7, 11000 Beograd, Serbia (Yugoslavia) \\ e-mail:aleks@ivic.matf.bg.ac.yu,aivic@rgf.rgf.bg.ac.yu
}

(Received 15 December, 1997)

\begin{abstract}
We investigate the function $R(T, \sigma)$, which denotes the error term in the asymptotic formula for $\int_{0}^{T}|\log \zeta(\sigma+i t)|^{2} d t$. It is shown that $R(T, \sigma)$ is uniformly bounded for $\sigma \geq 1$ and almost periodic in the sense of Bohr for fixed $\sigma \geq 1$; hence $R(T, \sigma)=\Omega(1)$ when $T \rightarrow \infty$. In case $\frac{1}{2}<\sigma<1$ is fixed we can obtain the bound $R(T, \sigma) \ll_{\varepsilon} T^{(9-2 \sigma) / 8+\varepsilon}$.
\end{abstract}

1991 Mathematics Subject Classification. Primary 11M06.

It is well known that the logarithm of the zeta-function may be written as the absolutely convergent Dirichlet series

$$
\log \zeta(s)=\log \prod_{p}\left(1-p^{-s}\right)^{-1}=\sum_{p} \log \left(1-p^{-s}\right)^{-1}=\sum_{k=1}^{\infty} \sum_{p} \frac{1}{k p^{k s}},
$$

where henceforth $p$ denotes a prime number. This expansion is valid in the halfplane $\operatorname{Re} s>1$, and therefore $\log \zeta(s)$ is almost periodic in this region. In particular, for $\sigma \geq 1$ and $-\infty<T<+\infty$, let

$$
\int_{0}^{T}|\log \zeta(\sigma+i t)|^{2} d t=T \sum_{k=1}^{\infty} \frac{1}{k^{2}} \sum_{p} \frac{1}{p^{2 k \sigma}}+R(T, \sigma) .
$$

The function $R(T, \sigma)$ may be thought of as the error term in the asymptotic formula (2), and it is classical that $R(T, \sigma)=o(T)$ as $|T|$ grows to infinity, for every fixed $\sigma>1$.

The behaviour of $\log \zeta$ is much more mysterious when $s$ lies to the left of the vertical line $\operatorname{Re} s=1$. For instance, the Riemann hypothesis is obviously equivalent to $\log \zeta$ being holomorphic in the quarter-plane $\operatorname{Re} s>\frac{1}{2}, \operatorname{Im} s>0$, and it is an important task to investigate this function there. A natural question is: does $\log \zeta$ retain some kind of almost-periodicity in the half-plane $\operatorname{Re} s>\frac{1}{2}$, in spite of possible singularities due to zeros of $\zeta$ (and of course the pole $s=1$ )? This is, for instance, dealt with in an important paper by Borchsenius and Jessen [4]. Our contribution to this topic is the study of the mean-square of $\log \zeta$ on vertical lines in this half-plane.

We may consider only the values $T>0$, since $\overline{\log \zeta(s)}=\log \zeta(\bar{s})$. Curiously we were not able to find anything relevant in the literature concerning the estimation of $R(T, \sigma)$; (nevertheless, see A. Selberg [13], where the mean square of $\arg \zeta\left(\frac{1}{2}+i t\right)$ is evaluated as well as moments of even order, A. Fujii [6], where the mean square of 
$\arg \zeta(\sigma+i(t+h))-\arg \zeta(\sigma+i t)$ is evaluated for $\frac{1}{2}<\sigma \leq 1$ and F.T. Wang [16] for a related problem). Our most precise result concerns the behaviour of $R(T, \sigma)$ in the case in which $\sigma \geq 1$ is fixed, and is contained in the following theorem.

Theorem 1. The function $R(T, \sigma)$ is uniformly bounded for $\sigma \geq 1$ and $-\infty<T<+\infty$. Moreover, if $\sigma \geq 1$ is fixed, then the function $R(T, \sigma)$ is almost periodic in the sense of $H$. Bohr.

Corollary. If $\sigma \geq 1$ is fixed, then the function $R(T, \sigma)$ has no limit when $T \rightarrow \infty$; hence $R(T, \sigma)=\Omega(1)$.

The corollary is an easy consequence of the theorem and the fact that $R(T, \sigma)$ is not a constant (because $|\log \zeta(\sigma+i t)|$ is not a constant). For the theory of almost periodic functions, we refer the reader to the book of $\mathrm{H}$. Bohr [3]. One may wonder if $R(T, \sigma)$ is uniformly almost periodic with respect to $\sigma \geq 1$. We cannot answer this question.

As we shall see, the proof of Theorem 1 follows from an application of Hilbert's inequality (in the version of Montgomery-Vaughan [12]) and the convergence of the series

$$
\sum_{n=1}^{\infty} \frac{1}{p_{n}\left(p_{n+1}-p_{n}\right)}
$$

where $p_{n}$ is the $n$-th prime.

Let us note that the definition of $R(T, \sigma)$ makes sense also if $\frac{1}{2}<\sigma<1$. The double series in (2) is clearly convergent in this range. Furthermore $\log \zeta(s)$ is analytic in the complex plane from which the countable union of half-lines $\left(s_{k}-\infty, s_{k}\right.$ ] has been removed, where $s_{k}$ runs through the zeros of $\zeta(s)$, or $s_{k}=1$. Further, when $\sigma$ is fixed, the function $\log \zeta(\sigma+i t)$ has discontinuities of the first kind or, at worst, logarithmic singularities, and so $R(T, \sigma)$ is a well defined function of $T$ for $\sigma>\frac{1}{2}$ fixed. However, if $\frac{1}{2}<\sigma<1$ we are unable to obtain results as sharp as when $\sigma \geq 1$, and in this case we shall prove a weaker result, given by the following theorem.

Theorem 2. Let $\frac{1}{2}<\sigma<1$ be a fixed number. For every positive $\varepsilon$, we have

$$
R(T, \sigma) \ll_{\varepsilon} T^{\frac{9-2 \sigma}{8}+\varepsilon} .
$$

We remark that our theorems could be generalized to the mean square of $\log F(s)$ for suitable Dirichlet series in the ranges $\sigma \geq \sigma_{a}$ and $\sigma>\alpha$, respectively, where $\sigma_{a}$ is the abscissa of absolute convergence of $F(s)$, and $\operatorname{Re} s=\alpha$ is the "critical line" for $F(s)$. An example of such a class of Dirichlet series is given by A. Selberg [14]. His Theorem 1 on p. 373, specialised to the case $F(s) \equiv \zeta(s)$, leads to the asymptotic formula

$$
\int_{0}^{T}\left|\log \zeta\left(\frac{1}{2}+i t\right)\right|^{2} d t=T \log \log T+O(T \sqrt{\log \log T}) .
$$

Finally if one wishes to evaluate the left-hand side of (2) for $\sigma<\frac{1}{2}$, then this case can be reduced to the case $\sigma>\frac{1}{2}$ by the use of the functional equation 


$$
\zeta(s)=\chi(s) \zeta(1-s), \quad \chi(s)=2^{s} \pi^{s-1} \sin \left(\frac{\pi s}{2}\right) \Gamma(1-s) .
$$

Proof of Theorem 1 . We begin by proving that $R(T, \sigma)$ is uniformly bounded for $\sigma \geq 1$ and $-\infty<T<+\infty$. Simple continuity and parity arguments show that it enough to consider the case $\sigma>1$ and $T \geq 0$. By (1) we have

$$
\log \zeta(\sigma+i t)=\sum_{k=1}^{\infty} \sum_{p} \frac{1}{k p^{k(\sigma+i t)}} .
$$

In order to evaluate the integral of $|\log \zeta(\sigma+i t)|^{2}$, we use the following lemma, due to Montgomery and Vaughan (see [11, (28) p. 140] and [12]).

Lemma 1. Let $\left\{a_{n}\right\}_{n=1}^{\infty}$ be a sequence of complex numbers and $\left\{\lambda_{n}\right\}_{n=1}^{\infty}$ a sequence of real numbers such that $\sum_{n=1}^{\infty}\left|a_{n}\right|<+\infty$ and $\theta_{n}:=\inf _{m \neq n}\left|\lambda_{m}-\lambda_{n}\right|>0$, for every $n$. Then

$$
\int_{0}^{T}\left|\sum_{n=1}^{\infty} a_{n} e^{i \lambda_{n} t}\right|^{2} d t=T \sum_{n=1}^{\infty}\left|a_{n}\right|^{2}+O\left(\sum_{n=1}^{\infty} \frac{\left|a_{n}\right|^{2}}{\theta_{n}}\right),
$$

where the $O$-constant is absolute.

We remark that the theorem of Montgomery and Vaughan is formulated for finite sums, but the hypotheses made in Lemma 1 permit this straightforward generalization. Hence

$$
R(T, \sigma) \ll \sum_{k=1}^{\infty} \sum_{p} \frac{1}{k^{2} p^{2 k} \delta(p, k)},
$$

where ( $p, q$ denote primes)

$$
\delta(p, k)=\min _{q^{\ell} \neq p^{k}}\left|\log q^{\ell}-\log p^{k}\right| .
$$

On the right-hand side of (5) the terms with $k \geq 2$ will be obviously convergent. When $k=1,(6)$ gives ( $p=p_{n}$, the $n$-th prime)

$$
\begin{aligned}
\delta\left(p_{n}, 1\right) & =\min _{q^{\ell}, \ell \geq 1}\left|\log q^{\ell}-\log p_{n}\right| \\
& =\min _{q^{\ell}, \ell \geq 1}\left|\log \frac{\left(q^{\ell}-p_{n}\right)+p_{n}}{p^{n}}\right| \gg \frac{\left|r_{n}-p_{n}\right|}{p_{n}},
\end{aligned}
$$

where $r_{n}=q^{\ell}$ is the prime power closest to $p_{n}$. Hence we have to estimate

$$
\sum_{n=1}^{\infty} \frac{1}{p_{n}\left|r_{n}-p_{n}\right|}
$$

Observing that $p_{n}\left|r_{n}-p_{n}\right| \gg r_{n}$, we see that the portion of the sum in (7) for which $r_{n}$ is not a prime is clearly convergent, and the remaining portion is convergent by 
the convergence of (3). In fact the convergence of (3) follows from the upper bound in the following estimate:

$$
\frac{x}{\log ^{2} x} \ll S(x):=\sum_{p_{n} \leq x} \frac{1}{p_{n+1}-p_{n}} \ll \frac{x \log \log x}{\log ^{2} x} .
$$

The lower bound in (8) follows easily from the Cauchy-Schwarz inequality; namely

$$
\frac{x}{\log x} \sim \sum_{p_{n} \leq x} 1 \leq\left(\sum_{p_{n} \leq x}\left(p_{n+1}-p_{n}\right) \cdot S(x)\right)^{1 / 2} \ll(x S(x))^{1 / 2} .
$$

For the upper bound we need a sieve estimate (see e.g. Halberstam-Richert [6]). We have that

$$
\sum_{p \leq x, p-h=p^{\prime}} 1 \ll \prod_{p \mid h}\left(1-\frac{1}{p}\right)^{-1} \frac{x}{\log ^{2} x}
$$

Thus, using this estimate we have

$$
\begin{aligned}
S(x) & =\sum_{h \leq H} \frac{1}{h} \sum_{p_{n} \leq x, p_{n+1}-p_{n}=h} 1+\sum_{h>H} \frac{1}{h} \sum_{p_{n} \leq x, p_{n+1}-p_{n}=h} 1 \\
& \ll \sum_{h \leq H} \frac{1}{h} \sum_{p \leq x, p-h=p^{\prime}} 1+\frac{x}{H \log x} \\
& \ll \sum_{h \leq H} \frac{1}{h \prod_{p \mid h}\left(1-\frac{1}{p}\right)} \cdot \frac{x}{\log ^{2} x}+\frac{x}{H \log x} \\
& =\sum_{h \leq H} \frac{1}{\varphi(h)} \cdot \frac{x}{\log ^{2} x}+\frac{x}{H \log x} \ll \frac{x \log H}{\log ^{2} x}+\frac{x}{H \log x} \ll \frac{x \log \log x}{\log ^{2} x}
\end{aligned}
$$

with the choice $H=\log x$. The bounds in (8) are given as (38)-(39) on p. 123 of Erdös-Rényi [5], but our proof is different from theirs. Nothing better than (8) seems to be known, and so improving (8) seems to be another interesting problem, which is indirectly related to the mean square of $\log \zeta(\sigma+i t)$.

Now we turn to the proof of the second part of the theorem. We consider separately the cases $\sigma>1$ and $\sigma=1$. In the first case, the function $R(T, \sigma)$ is the primitive of a function almost periodic in the sense of Bohr, and the fact that it is bounded ensures its Bohr almost-periodicity, according to a well known theorem of Bohr (see [2, p. 123]). In the second case, the function $|\log \zeta(1+i t)|^{2}$ is no longer Bohr almost-periodic (in fact, it is not even bounded). Nevertheless this function is almost periodic in the sense of Stepanoff $\left(L^{1}\right)$ and the theorem of Bohr mentioned above is still valid in this case, as is easily seen by inspection of the proof; (for the definition and main properties of almost periodicity in the sense of Stepanoff, see [15]; Bochner [1] is also relevant, especially p. 251). To see that $|\log \zeta(1+i t)|^{2}$ is almost periodic in the sense of Stepanoff $\left(L^{1}\right)$, it suffices to prove that $\log \zeta(1+i t)$ is almost periodic in the sense of Stepanoff $\left(L^{2}\right)$. This last fact is a consequence of the 
following lemma, essentially due to Wiener and Wintner (cf. [17]) and suggested to us by the reading of a recent paper of J.-P. Kahane [10].

Lemma 2. Let $F(s)=\sum_{n=1}^{\infty} a_{n} n^{-s}$ be a Dirichlet series with nonnegative coefficients $\left(a_{n} \geq 0\right)$ and abscissa of convergence 1 . Let $\delta>0$ be such that $F(\sigma+i t)$ has a limit in $L^{2}(-\delta, \delta)$ when $\sigma \rightarrow 1$. Then $F(\sigma+i t)$ has a limit in the Stepanoff $L^{2}$-metric when $\sigma \rightarrow 1$.

Proof of Lemma 2. Recall that the Stepanoff $L^{2}$-norm of a function $f$ is given by

$$
\|f\|_{S}^{2}=\sup _{-\infty<x<+\infty} \int_{x}^{x+1}|f(t)|^{2} d t .
$$

Suppose first that $\delta=\frac{1}{2}$. By the completeness of the Stepanoff norm, it is enough to prove that

$$
\int_{x-\frac{1}{2}}^{x+\frac{1}{2}}\left|F\left(\sigma_{1}+i t\right)-F\left(\sigma_{2}+i t\right)\right|^{2} d t
$$

can be made arbitrarily small if $1<\sigma_{1}<\sigma_{2}<1+\alpha$, where $\alpha$ is small enough, uniformly in $x$. Due to the nonnegativity of the $a_{n}$ 's and an estimate of H.L. Montgomery ([11, Theorem 3, p. 131]), this integral is less than

$$
3 \int_{-\frac{1}{2}}^{\frac{1}{2}}\left|F\left(\sigma_{1}+i t\right)-F\left(\sigma_{2}+i t\right)\right|^{2} d t,
$$

and the result follows immediately. If $\delta \neq \frac{1}{2}$, we obtain the same result for the metric

$$
\|f\|_{S, \delta}^{2}=\sup _{-\infty<x<+\infty}(2 \delta)^{-1} \int_{x-\delta}^{x+\delta}|f(t)|^{2} d t,
$$

which is known to be equivalent to the one with $\delta=\frac{1}{2}$.

Now the fact that $\log \zeta(\sigma+i t)$ converges in $L^{2}\left(-\frac{1}{2}, \frac{1}{2}\right)$, say, follows by the analyticity of $\zeta(s)$ and the Lebesgue dominated convergence theorem. The function $\log \zeta(1+i t)$ is thus almost periodic in the sense of Stepanoff $\left(L^{2}\right)$, as a Stepanofflimit of Bohr almost-periodic functions, as was to be shown.

Proof of Theorem 2. The method that we shall use bears analogies to the one employed in [9] to investigate large values of $\zeta(s)$ near the line $\sigma=1$. Suppose that $\frac{1}{2}<\sigma<1$ is fixed and $T \geq T_{0}>0$. Choose $\delta$ such that $\frac{1}{2}<\delta<\sigma$, and let

$$
[T, 2 T]=\mathcal{A}(T) \cup \mathcal{B}(T),
$$

where $\mathcal{A}(T)$ consists of $t \in[T, 2 T]$ such that $\zeta(w) \neq 0$ for $\operatorname{Re} w \geq \delta$, $|\operatorname{Im} w-t| \leq \log ^{2} T$. We start from the Mellin inversion integral (see (A.7) of [8])

$$
e^{-x}=\frac{1}{2 \pi i} \int_{c-i \infty}^{c+i \infty} x^{-s} \Gamma(s) d s \quad(c, x>0)
$$


and the Dirichlet series representation (1). For $T \leq t \leq 2 T, s=\sigma+i$ and $X=T^{A}$ with suitable $A>0$ we have that

$$
\frac{1}{2 \pi i} \int_{2-i \infty}^{2+i \infty} \log \zeta(s+w) X^{w} \Gamma(w) d w=\sum_{k=1}^{\infty} \sum_{p} \frac{1}{k p^{k s}} \exp \left(-\frac{p^{k}}{X}\right) .
$$

Let $t \in \mathcal{A}(T)$, choose $\alpha$ such that $\delta<\alpha<\sigma$, and replace the line of integration in (9) by the contour $\mathcal{L}$ consisting of $\left[\alpha-\sigma-\frac{i}{2} \log ^{2} T, \alpha-\sigma+\frac{i}{2} \log ^{2} T\right]$, $\left[\alpha-\sigma \pm \frac{i}{2} \log ^{2} T, 2 \pm \frac{i}{2} \log ^{2} T\right],\left[2 \pm \frac{i}{2} \log ^{2} T, 2 \pm i \infty\right)$. Then the integrand will be regular on $\mathcal{L}$, and the only pole of the integrand that is passed is $w=0$, which is a simple pole with residue $\log \zeta(s)$. Note that the singularity $w=1-s$ lies to the left of $\mathcal{L}$, and thus need not be considered. We have

$$
\log \zeta(s+w) \ll \log T \quad(w \in \mathcal{L})
$$

This follows by integration from the formula (see (1.52) of [8] or (2.9) of [9])

$$
\frac{\zeta^{\prime}(s)}{\zeta(s)}=\sum_{\rho,|\operatorname{Im} \rho-t| \leq 1} \frac{1}{s-\rho}+O(\log t)
$$

where $\zeta(\rho)=0, s=\sigma+i t,-1 \leq \sigma \leq 2, t \geq t_{0}>0$. Thus using (10) and Stirling's formula for the gamma-function we obtain

$$
\frac{1}{2 \pi i} \int_{\mathcal{L}} \log \zeta(s+w) X^{w} \Gamma(w) d w \ll X^{\alpha-\sigma} \log T .
$$

It then follows from (9) and the residue theorem that

$$
\log \zeta(s)=\sum_{n=1}^{\infty} a_{n} n^{-i t}+O\left(X^{\alpha-\sigma} \log T\right) \quad(t \in \mathcal{A}(T)),
$$

where $a_{n}=\frac{1}{k} p^{-k \sigma} \exp \left(-\frac{p^{k}}{X}\right)$ if $n=p^{k}$, and $a_{n}=0$ otherwise. We have

$$
\int_{T}^{2 T}|\log \zeta(\sigma+i t)|^{2} d t=\left(\int_{\mathcal{A}(T)}+\int_{\mathcal{B}(T)}\right)|\log \zeta(\sigma+i t)|^{2} d t=I_{1}+I_{2},
$$

say. To evaluate $I_{1}$ we shall apply the mean value theorem for Dirichlet polynomials in the form given by Lemma 1 . We obtain

$$
\int_{T}^{2 T}\left|\sum_{n=1}^{\infty} a_{n} n^{-i t}\right|^{2} d t=T \sum_{n=1}^{\infty}\left|a_{n}\right|^{2}+O\left(\sum_{n=1}^{\infty} n\left|a_{n}\right|^{2}\right)
$$

We have 
THE LOGARITHM OF THE ZETA-FUNCTION

$$
\begin{aligned}
\sum_{n=1}^{\infty}\left|a_{n}\right|^{2} & =\sum_{k=1}^{\infty} \frac{1}{k^{2}} \sum_{p} p^{-2 k \sigma} \exp \left(-\frac{2 p^{k}}{X}\right) \\
& =\sum_{k=1}^{\infty} \frac{1}{k^{2}} \sum_{p \leq X^{1 / 2 k)}} p^{-2 k \sigma} \exp \left(-\frac{2 p^{k}}{X}\right)+O\left(X^{\frac{1}{2}-\sigma}\right) \\
& =\sum_{k=1}^{\infty} \frac{1}{k^{2}} \sum_{p \leq X^{1 /(2 k)}} p^{-2 k \sigma}\left(1+O\left(\frac{p^{k}}{X}\right)\right)+O\left(X^{\frac{1}{2}-\sigma}\right) \\
& =\sum_{k=1}^{\infty} \frac{1}{k^{2}} \sum_{p} p^{-2 k \sigma}+O\left(X^{\frac{1}{2}-\sigma}\right)
\end{aligned}
$$

and

$$
\sum_{n=1}^{\infty} n\left|a_{n}\right|^{2}=\sum_{k=1}^{\infty} \frac{1}{k^{2}} \sum_{p \leq X \log X} p^{k(1-2 \sigma)} \exp \left(-\frac{2 p^{k}}{X}\right)+O(1) \ll X^{2-2 \sigma}
$$

It follows that

$$
\int_{T}^{2 T}\left|\sum_{n=1}^{\infty} a_{n} n^{-i t}\right| d t \leq\left(T \int_{T}^{2 T}\left|\sum_{n=1}^{\infty} a_{n} n^{-i t}\right|^{2} d t\right)^{1 / 2} \ll T+T^{\frac{1}{2}} X^{1-\sigma} .
$$

Hence, in view of $|a+b|^{2}=|a|^{2}+|b|^{2}+2 \operatorname{Re} \bar{a} b$, (12) yields

$$
\begin{aligned}
& I_{1}=\int_{\mathcal{A}(T)}\left|\sum_{n=1}^{\infty} a_{n} n^{-i t}\right|^{2} d t+O\left(T X^{\alpha-\sigma} \log T\right) \\
& =\int_{T}^{2 T}\left|\sum_{n=1}^{\infty} a_{n} n^{-i t}\right|^{2} d t-\int_{\mathcal{B}(T)}\left|\sum_{n=1}^{\infty} a_{n} n^{-i t}\right|^{2} d t+O\left(T X^{\alpha-\sigma} \log T\right),
\end{aligned}
$$

provided that

$$
X \leq T^{1 /(2-2 \sigma)}
$$

If $N(\sigma, T)$ denotes the number of complex zeros $\rho=\beta+i \gamma$ of $\zeta(s)$ such that $\beta \geq \sigma,|\gamma| \leq T$ and $\mu(\cdot)$ denotes Lebesgue linear measure, then

$$
\mu(\mathcal{B}(T)) \ll N\left(\delta, T+\log ^{2} T\right) \log ^{2} T \ll T^{\frac{3-3 \delta}{2-\delta}} \log ^{7} T \ll T^{\frac{3}{2}-\delta} \log ^{7} T,
$$

where we used the classical bound of A.E. Ingham for $N(\sigma, T)$. See (11.26) of [8]. The crucial fact is that the exponent of $T$ in (15) is less than 1 . We obtain

$$
\begin{aligned}
& \int_{\mathcal{B}(T)}\left|\sum_{n=1}^{\infty} a_{n} n^{-i t}\right|^{2} d t=\int_{\mathcal{B}(T)}\left|\sum_{n \leq X \log ^{2} X} a_{n} n^{-i t}\right|^{2} d t+O(1) \\
& \leq\left(\int_{\mathcal{B}(T)} 1 d t\right)^{1 / 2}\left(\int_{\mathcal{B}(T)}\left|\sum_{n \leq X \log ^{2} X} a_{n} n^{-i t}\right|^{4} d t\right)^{1 / 2}+O(1) \\
& \ll \mu^{1 / 2}(\mathcal{B}(T))\left(\int_{T}^{2 T}\left|\sum_{n \leq X^{2} \log ^{4} X} c_{n} n^{-\sigma-i t}\right|^{2} d t\right)^{1 / 2}+1
\end{aligned}
$$


with $0 \leq c_{n} \leq d(n)$, where $d(n)$ is the number of divisors of $\mathrm{n}$. Applying again the mean value theorem for Dirichlet polynomials we see that the last integral above is

$$
\begin{aligned}
& \ll T+\sum_{n \leq X^{2} \log ^{4} X} d^{2}(n) n^{1-2 \sigma} \\
& \ll T+\left(X^{2} \log ^{4} X\right)^{2-2 \sigma} \log ^{3} X \ll T+X^{4-4 \sigma} \log ^{7} T .
\end{aligned}
$$

Thus we obtain

$$
\int_{\mathcal{B}(T)}\left|\sum_{n=1}^{\infty} a_{n} n^{-i t}\right|^{2} d t \ll T^{\frac{3}{4}-\frac{\delta}{2}}\left(T^{\frac{1}{2}}+X^{2-2 \sigma}\right) \log ^{7} T
$$

Consequently from (13) and (16) we obtain, provided that (14) holds,

$$
\begin{aligned}
I_{1} & =T \sum_{k=1}^{\infty} \frac{1}{k^{2}} \sum_{p} p^{-2 k \sigma} \\
& +O\left(T X^{\frac{1}{2}-\sigma}+T X^{\alpha-\sigma} \log T+T^{\frac{3}{4}-\frac{\delta}{2}}\left(T^{\frac{1}{2}}+X^{2-2 \sigma}\right) \log ^{7} T\right) .
\end{aligned}
$$

It remains to estimate

$$
\begin{aligned}
I_{2} & =\int_{\mathcal{B}(T)}|\log \zeta(\sigma+i t)|^{2} d t \ll \int_{\mathcal{B}(T)}\left(\log ^{2}|\zeta(\sigma+i t)|+\arg ^{2} \zeta(\sigma+i t)\right) d t \\
& \ll \int_{\mathcal{B}(T)} \log ^{2}|\zeta(\sigma+i t)| d t+T^{\frac{3}{2}-\delta} \log ^{9} T
\end{aligned}
$$

since $\arg \zeta(\sigma+i t) \ll \log t$. We use the formula

$$
\log |\zeta(s)|=\sum_{\rho,|t-\gamma| \leq 1} \log |s-\rho|+O(\log t) \quad(\rho=\beta+i \gamma, s \neq \rho,-1 \leq \sigma \leq 2) .
$$

One obtains (19) by integrating (11) over $[\sigma+i t, 2+i t]$ and then taking real parts of the resulting expression. Moreover the contribution of $\rho=\beta+i \gamma$ in (19) such that $\beta<\delta$ is $\ll \log t$. Then the last integral in (18) is, by the Cauchy-Schwarz inequality,

$$
\ll I_{3} \log T+T^{\frac{3}{2}-\delta} \log ^{9} T
$$

with

$$
\begin{aligned}
I_{3} & :=\int_{\mathcal{B}(T)} \sum_{|t-\gamma| \leq 1, \beta \geq \delta} \log ^{2}|s-\rho| d t \\
& \leq \sum_{T-1 \leq \gamma \leq 2 T+1, \beta \geq \delta} \int_{\mathcal{B}(T) \cap[\gamma-1, \gamma+1]} \log ^{2}|t-\gamma| d t \\
& \leq N(\delta, 2 T+1) \int_{-1}^{1} \log ^{2}|u| d u \ll T^{\frac{3}{2}-\delta} \log ^{5} T .
\end{aligned}
$$


Therefore it follows that

$$
I_{2} \ll T^{\frac{3}{2}-\delta} \log ^{9} T
$$

Finally from (17) and (20), given that $\frac{1}{2}<\delta<\alpha<\sigma<1$ we have

$$
\begin{aligned}
& R(2 T, \sigma)-R(T, \sigma) \\
& \ll\left(T X^{\alpha-\sigma}+T^{\frac{5}{4}-\frac{\delta}{2}}+T^{\frac{3}{4}-\frac{\delta}{2}} X^{2-2 \sigma}\right) \log ^{9} T \ll_{\varepsilon} T^{\frac{9-2 \sigma}{8}+2 \varepsilon},
\end{aligned}
$$

if we choose $X=T^{1 / 2}$ (so that (14) holds), $\delta=\frac{\sigma}{2}+\frac{1}{4}-2 \varepsilon, \alpha=\frac{\sigma}{2}+\frac{1}{4}+2 \varepsilon$. Replacing $T$ by $T / 2, T / 2^{2}, \ldots, \varepsilon$ by $\frac{1}{2}$ and adding the resulting estimates we obtain the assertion of the theorem.

The optimal value of the exponent of $T$ in Theorem 2 is not easy to determine, since it depends on bounds for the zero-density function $N(\sigma, T)$ (see Chapter 11 of [8] for a discussion concerning bounds for $N(\sigma, T)$ ). If the Riemann hypothesis (that all complex zeros of $\zeta(s)$ have real parts equal to $\frac{1}{2}$ ) holds, then the above discussion may be considerably simplified. Taking $\alpha=\frac{1+\varepsilon}{2}(0<\varepsilon<2 \sigma-1)$ we obtain

$$
R(2 T, \sigma)-R(T, \sigma) \ll_{\varepsilon} T^{\varepsilon}\left(T X^{\frac{1}{2}-\sigma}+T^{\frac{1}{2}} X^{\frac{3}{2}-2 \sigma}+X^{2-2 \sigma}\right) .
$$

Hence choosing $X=T^{2 /(3-2 \sigma)}$ we obtain, on the Riemann hypothesis,

$$
R(T, \sigma) \ll_{\varepsilon} T^{\frac{4-4 \sigma}{3-2 \sigma}+\varepsilon} .
$$

Note that we have shown that $R(T, \sigma)=\Omega(1)$ as $T \rightarrow \infty$ for $\sigma \geq 1$ fixed. It would be interesting to obtain an $\Omega$-result for $R(T, \sigma)$ when $\frac{1}{2}<\sigma<1$ is fixed, which would perhaps give some indication as to what the true order of magnitude of $R(T, \sigma)$ might be.

\section{REFERENCES}

1. S. Bochner, Remark on the integration of almost periodic functions, J. London Math. Soc. 8 (1933), 250-254. 127.

2. H. Bohr, Zur Theorie der fastperiodischen Funktionen, I, Acta Math. 45 (1925), 29-

3. H. Bohr, Almost periodic functions (Chelsea, New York, 1951).

4. V. Borchsenius and B. Jessen, Mean motions and values of the Riemann zeta function, Acta Math. 80 (1948), 97-166.

5. P. Erdös and A. Rényi, Some problems and results on consecutive primes, Simon Stevin 27 (1950), 115-125.

6. A. Fujii, Number variance of the zeros of the Epstein zeta functions, Proc. Japan Acad. Ser. A Math. Sci. 70 (1994), 140-145.

7. H. Halberstam and H.-E. Richert, Sieve methods (Academic Press, 1974).

8. A. Ivić, The Riemann zeta-function (John Wiley \& Sons, 1985).

9. A. Ivić, Large values of some zeta-functions near the line $\sigma=1$, Hardy-Ramanujan J. 11 (1988), 13-29.

10. J.-P. Kahane, Sur les nombres premiers généralisés de Beurling. Preuve d'une conjecture de Bateman et Diamond, J. Théorie Nombres Bordeaux 9 (1997), 251-266.

11. H. L. Montgomery, Ten lectures on the interface between analytic number theory and harmonic analysis, CBMS Regional Conference Series in Mathematics, 84 (AMS, Providence, R.I., 1994). 
12. H. L. Montgomery and R. C. Vaughan, Hilbert's inequality, J. London Math. Soc. (2) 8 (1974), 73-82.

13. A. Selberg, Contributions to the theory of the Riemann zeta-function, Arch. Mat. Naturvid. 48 (1946), No. 5, 89-155.

14. A. Selberg, Old and new conjectures and results about a class of Dirichlet series, in Proceedings of the Amalfi Conference on Analytic Number Theory (eds. E. Bombieri et al.), (Universitá di Salerno, Salerno, 1992), 367-385.

15. W. Stepanoff, Über einige Verallgemeinerungen der fast periodischen Funktionen, Math. Ann. 95 (1926), 473-498.

16. F. T. Wang, A mean value theorem of the Riemann zeta-function, Quart. J. Math. Oxford Ser. 18 (1947), 1-3.

17. N. Wiener and A. Wintner, On a local $L^{2}$-variant of Ikehara's theorem, Rev. Math. Cuyana 2 (1956), 53-59. 